to be a major cause of morbidity and mortality among children and adults with SLE. Up to $60 \%$ of adult and $80 \%$ of pediatric SLE patients (pts) will eventually develop overt renal disease [1]. To date the excess burden of comorbidities, risk of inpatient hospitalization, and in-hospital death associated with SLE and LN remains incompletely understood.

Objectives: To identify differences in comorbidities, hospitalizations, and inhospital mortality of SLE and LN cohorts compared to: 1) each other; 2) reference populations of pts without an autoimmune condition (non-Al) matched on gender and age. Reference populations were allowed to have claims for non-autoimmune conditions.

Methods: We conducted a retrospective cohort study using the Truven Healthcare MarketScan ${ }^{\circledR}$ Commercial Claims and Encounters and Medicare Supplemental and Coordination of Benefits database, which together comprise 65 million insured US lives between 1999 and 2014. Cohort identification is based on validated algorithms [2,3] for identification of pts with either LN or SLE without renal involvement using claims data. Pts were matched on age and gender at index date. All eligible participants had 365 days of enrollment prior to and after the index date. End of study for post-index follow-up was captured as whichever of the following occurred first: 1) end of enrollment; 2) end of database; 3 ) date of death. Results are presented separately for pediatric and adult pts.

Results: 54,813 SLE pts without renal involvement and 8,839 LN pts were identified and matched to reference non Al populations. Compared to the nonrenal SLE cohort, pts in the LN cohort were older ( $49.9 \pm 16.6$ vs. $48.6 \pm 14.3$ years) with a higher proportion of males (15.4\% vs. $11.2 \%$ ). Pts with $L N$ had the highest scores on the Charlson Comorbidity Index modified to exclude renal involvement (Table 1). Additionally, adults with LN had higher rates of hospitalizations and longer hospitalizations compared with adults with non-renal SLE, who already had higher rates of hospitalizations and longer hospitalizations than matched controls (Table 2). This pattern of findings was consistent for children. Rates of in-hospital mortality were highest among those with LN but also increased among those with SLE compared with matched controls (Figure 1).

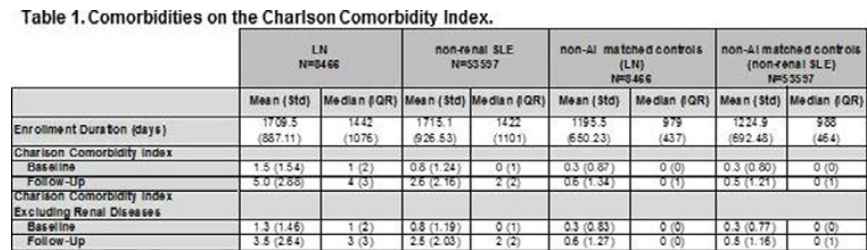

Table 2. Hospitalizations and mortality by age and patient population.

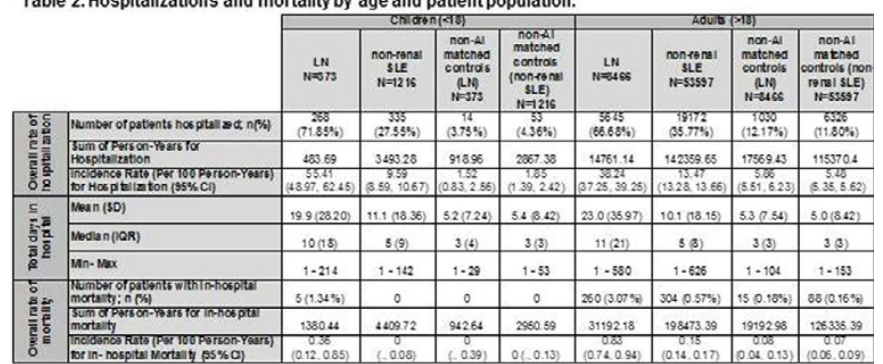

Flgure 1: Rates of in-hoepltal mortallty by group and age

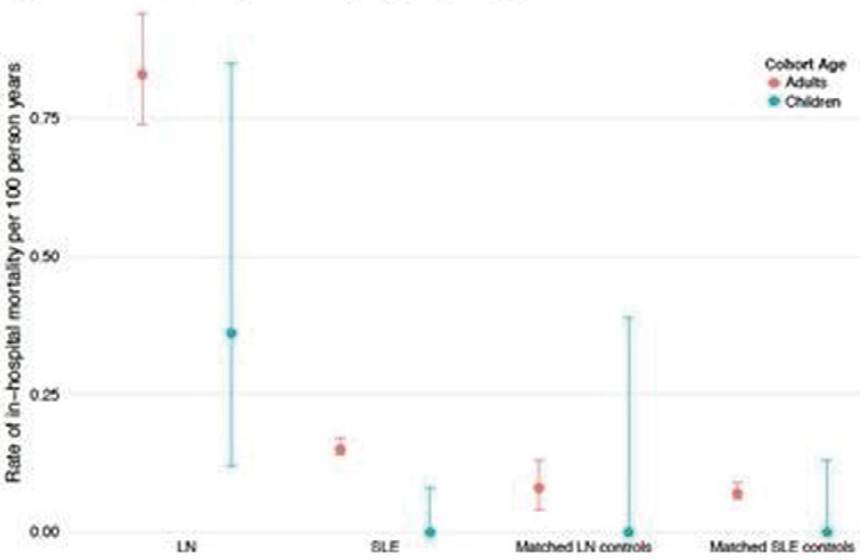

Conclusions: An SLE diagnosis was associated with a higher burden of comorbidities and higher rates of hospitalizations and in-hospital mortality than non-Al matched controls. Pts with $\mathrm{LN}$ had the highest burden of comorbidities and rates of hospitalizations and in-hospital mortality. SLE and LN impose a high burden of morbidity and mortality and the medical need for safe and effective treatments of LN and SLE remains unmet. Clinicians should consider these factors in their assessment and treatment of pts with SLE and LN. The retrospective, claims-based results do not permit pt-level assessment of the relative contributions of disease, treatment, and potential confounders to these findings.

References:

[1] Cameron JS. J Am Soc Nephrol, 1999:10:413-24.

[2] Arkema EV et al. BMJ open, 2016;6:e007769.

[3] Chibnik LB et al. Lupus, 2010;19:741-3.

Disclosure of Interest: K. Belendiuk Employee of: Genentech, Inc., H. Trinh Employee of: Genentech, Inc., M. Cascino Employee of: Genentech, Inc., L. Dragone Employee of: Genentech, Inc., D. Keebler Employee of: Genentech, Inc. J. Garg Employee of: Genentech, Inc.

DOI: 10.1136/annrheumdis-2017-eular.5526

\section{FRI0286 SEROLOGICAL EVOLUTION IN PATIENTS WITH THROMBOTIC ANTIPHOSPHOLIPID SYNDROME}

L. Riancho-Zarrabeitia ${ }^{1}$, S. García-Canale ${ }^{1}$, M. Cubería $^{1}$, G. Daroca ${ }^{1}$, M. Agudo ${ }^{1}$, M. López-Hoyos ${ }^{2}$, P. Muñoz ${ }^{1}$, V. Martínez-Taboada ${ }^{3} .{ }^{1}$ Hospital $^{\prime}$ Universitario Marqués de Valdecilla, Santander, Spain; ${ }^{2}$ Immunology;

${ }^{3}$ Rheumatology, Hospital Universitario Marqués de Valdecilla, Santander, Spain

Background: Antiphospholipid syndrome (APS) is an autoinmune disease characterized by the presence of antiphospholipid antibodies (aPL) and at least one clinical event (thrombosis and /or pregnancy morbidities). The titers of aPL can fluctuate and eventually become negative. This negativization, particularly if persistent, may be associated with a lower frequency of clinical events.

Objectives: To describe the clinical and serological course of patients with thrombotic APS as well as the factors related with the aPL negativization

Methods: We performed a retrospective study including patients attended at the Rheumatology clinic from a tertiary hospital in Northern Spain. We included 94 patients with thrombotic APS according to Sidney criteria of 2006. They were classified according to the serological evolution as persistently negative $\mathrm{aPL}$, transiently positive, and persistently positive aPL according to previously established criteria.

Results: After a mean follow-up of $145 \pm 56$ months, $48.9 \%$ of patients presented a persistently negative serology, whereas in $12.8 \%$ it was transiently positive, and persistently positive in $38.3 \%$. When analyzing potential factors related to the negativization (table 1), we found that patients with positive lupus anticoagulant tended to have a persistently negative serology during follow-up, but it did not reach statistical significance (OR 2.7; 95\% $\mathrm{Cl} 0.8-9.4 ; \mathrm{p}=0.145)$. We found no association between traditional cardiovascular risk factors or previous treatments and the serological evolution.

\begin{tabular}{lcccc}
\hline Variable & Total & $\begin{array}{c}\text { Persistently } \\
\text { negative } \\
(\mathrm{n}=46)\end{array}$ & $\begin{array}{c}\text { Persistently positive and } \\
\text { transiently positive } \\
(\mathrm{n}=48)\end{array}$ & $\mathrm{P}$ \\
\hline Age & $45 \pm 16$ & $44 \pm 14$ & $47 \pm 17$ & 0.33 \\
Male sex, $\mathrm{n}(\%)$ & $19(21)$ & $8(18)$ & $11(24)$ & 0.61 \\
SLE, $\mathrm{n}(\%)$ & $25(27)$ & $12(26)$ & $13(27)$ & 1 \\
Load of antibodies, $\mathrm{n}(\%)$ & & 0.94 & & \\
1 & $29(31)$ & $15(33)$ & $14(29)$ & \\
2 & $40(43)$ & $19(41)$ & $21(44)$ & \\
3 & $25(27)$ & $12(26)$ & $13(27)$ & \\
Anticardiolipin antibodies, $\mathrm{n}(\%)$ & $76(81)$ & $36(78)$ & $40(83)$ & 0.60 \\
Antip2-Glycoproteinl antibodies, $\mathrm{n}(\%)$ & $59(63)$ & $27(59)$ & $32(67)$ & 0.52 \\
Lupus anticoagulant, $\mathrm{n}(\%)$ & $35(66)$ & $18(78)$ & $17(57)$ & 0.14 \\
Family history of thrombosis, $\mathrm{n}(\%)$ & $17(31)$ & $10(40)$ & $7(24)$ & 0.25 \\
Tobacco use, $\mathrm{n}(\%)$ & $41(44)$ & $21(46)$ & $20(42)$ & 0.83 \\
Hypertension, $\mathrm{n}(\%)$ & $45(48)$ & $21(46)$ & $24(50)$ & 0.69 \\
Dyslipidemia, $\mathrm{n}(\%)$ & $43(46)$ & $21(46)$ & $22(46)$ & 1 \\
Diabetes, $\mathrm{n}(\%)$ & $4(4)$ & $2(4)$ & $2(4)$ & 1 \\
Antimalarials, $\mathrm{n}(\%)$ & $34(36)$ & $16(35)$ & $18(37)$ & 0.83 \\
Heparin, $\mathrm{n}(\%)$ & $34(36)$ & $16(35)$ & $18(37)$ & 0.83 \\
Oral anticoagulants, $\mathrm{n}(\%)$ & $68(72)$ & $33(72)$ & $35(73)$ & 1 \\
Antiplatelets, $\mathrm{n}(\%)$ & $71(76)$ & $33(73)$ & $38(79)$ & 0.63 \\
Corticosteroids, $\mathrm{n}(\%)$ & $5(5)$ & $2(4)$ & $3(6)$ & 1 \\
Immunosuppressants, $\mathrm{n}(\%)$ & $4(4)$ & $1(2)$ & $3(6)$ & 0.36 \\
\hline
\end{tabular}

Conclusions: After a mean follow-up of 12 years, $49 \%$ of thrombotic APS patients presented a persistently negative serology. We found no significant association between immunological, traditional cardiovascular risk factors or previous treatments and the persistently negative serology.

Disclosure of Interest: None declared

DOI: 10.1136/annrheumdis-2017-eular.5106

\section{FRI0287 INCIDENCE OF LUPUS NEPHRITIS AND 18-MONTH FOLLOW UP IN COLOMBIAN PATIENTS WITH SYSTEMIC LUPUS ERYTHEMATOSUS}

M.A. Alzate ${ }^{1}$, D. Hernandez-Parra ${ }^{1}$, J.C. Salazar-Uribe ${ }^{2}$, P. Ortiz-Salazar ${ }^{1}$, R. Pineda ${ }^{1}$ on behalf of Artmedica IPS, Medellin, Colombia. ${ }^{1}$ Clinical information group, Artmedica IPS; ${ }^{2}$ School of Statistics, Faculty of Sciences, National University of Colombia, Medellin, Colombia

Background: Lupus nephritis (LN) is one of the major indicators of poor prognosis in patients with systemic lupus erythematosus (SLE). Multiple studies with Latin- 
American SLE population have shown a higher prevalence of LN, higher severity, and less favorable outcomes (1).

Objectives: To determine the incidence of lupus nephritis and end-stage renal failure, as well as evaluate progression of renal function and proteinuria during an 18-month follow up in colombian patients with SLE.

Methods: A retrospective cohort study was conducted in 1448 patients with SLE, 41 of which were diagnosed with LN between August/2014 and July/2015. Follow up was made for 18 months, analyzing glomerular filtration rate (GFR) and proteinuria, induction and manteinance therapy, renal relapses, hospitalizations and mortality. Univariate analysis was done to describe sociodemographic and clinical variables. Longitudinal data analysis was performed using linear mixed models with random intercepts. In all cases, a $\mathrm{p}$ value $<0.05$ was considered statistically significant.

Results: Clinical characteristics of patients with LN are shown in table 1. Eightyfive percent of $L N s$ where biopsy-proven. Incidence of $L N$ was 2,83 cases/100 SLE patients/year. The incidence of end-stage renal failure was 7,31 cases/100 LN patients. During the 18 -month follow up, $34 \%$ of patients had hospitalizations related to SLE activity or complications, $7,3 \%$ renal relapse, $2,4 \%$ rebiopsy, and no mortality cases. Induction therapy was done with cyclophosphamide in $58,5 \%$ and with mycophenolate mofetil in $41,4 \%$, with $30 \%$ of the patients requiring re-induction therapy, Ten percent of patients required use of rituximab due to refractory response to multiple treatments. In longitudinal linear analyses, age at

Table 1. Clinical characteristics of patients with lupus nephritis

\begin{tabular}{lc}
\hline Characteristic (N=41) & Mean (SD) \\
\hline Age & $37,2(11,2)$ \\
Age at diagnosis & $32,2(11,1)$ \\
Years of evolution & $4,6(4,4)$ \\
& $\mathrm{N}(\%)$ \\
Female & $38(92,7)$ \\
Malar rash & $11(28,2)$ \\
Discoid lupus & $2(4,9)$ \\
Photosensitivity & $18(46,1)$ \\
Oral ulcers & $14(34,1)$ \\
Serositis & $16(44,4)$ \\
Arthritis & $31(75,6)$ \\
Neurological involvement & $5(12,2)$ \\
Hematological involvement & $32(78)$ \\
ANAs $(+)$ & $39 / 39(100)$ \\
Anti dsDNA $(+)$ & $29 / 41(70,7)$ \\
Anti Sm $(+)$ & $17 / 35(48,6)$ \\
Anti Ro $(+)$ & $19 / 36(52,7)$ \\
Anti La $(+)$ & $10 / 34(29,4)$ \\
Anti RNP $(+)$ & $18 / 36(50)$ \\
\hline
\end{tabular}

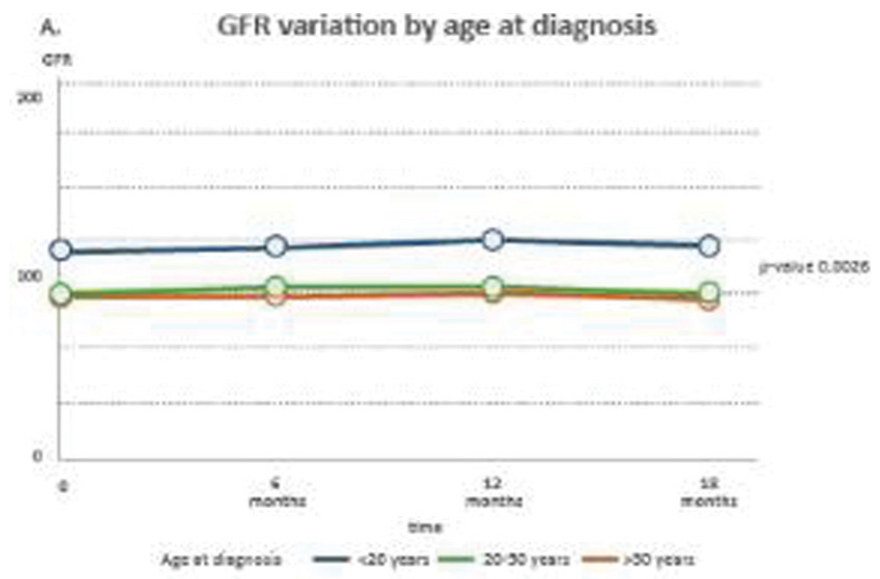

B. Proteinuria variation by biopsy classification

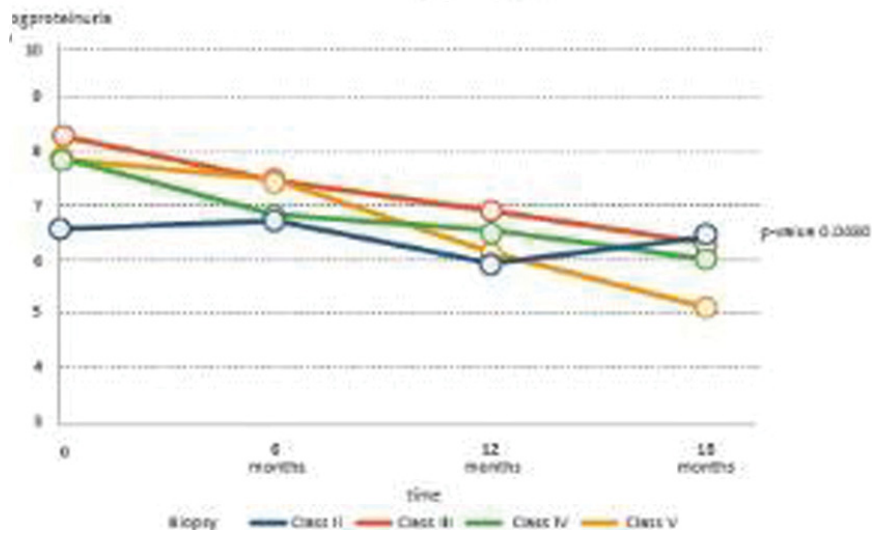

diagnosis and anti dsDNA was positively associated to GFR variations (Figure $1, \mathrm{~A})$, while anti Sm, hematologic involvement and biopsy classification were associated to proteinuria variations during time of follow-up (Figure 1,B).

Conclusions: In a real life scenario, anual incidence of lupus nephritis was 2,83 cases/100 SLE patients. A high proportion of patients with refractory response to multiple immunomodulatory treatments for $L N$ were identified.

References:

[1] Pons-Estel GJ, Catoggio LJ, Cardiel MH, Bonfa E, Caeiro F, Sato E, et al. Lupus in Latin-American patients: lessons from the GLADEL cohort. Lupus. 2015;24(6):536-45.

Disclosure of Interest: None declared

DOI: 10.1136/annrheumdis-2017-eular.6137

\section{FRI0288 NAILFOLD CAPILLAROSCOPIC PICTURES IN A COHORT OF UNDIFFERENTIATED CONNECTIVE TISSUE DISEASE (UCTD) PATIENTS AND IN THOSE THAT MOVE TO SYSTEMIC LUPUS ERYTHEMATOSUS}

M. Meroni ${ }^{1}, 2$, C. Pizzorni ${ }^{1}$, A. Sulli ${ }^{1}$, M. Limonta ${ }^{2}$, M. Cutolo ${ }^{1} .{ }^{1}$ Research Laboratory and Academic Division of Clinical Rheumatology, Internal Medicine Dept., University of Genova, Genova; ${ }^{2}$ Rheumatology Unit, ASST Papa Giovanni XXIII, Bergamo, Italy

Background: Nailfold capillaroscopy (NVC) is a useful, non-invasive, reproducible and cost-effective diagnostic tool, able to assess the shape of capillaries in the nailfold bed. According to the presence of peculiar abnormalities, it is essential in the early differential diagnosis of connective tissue diseases (CTDs), mainly "scleroderma-spectrum disorders" (SSD). Despite its large diffusion, no univocal NVC patterns have been ascribed to undifferentiated connective tissue disease (UCTD) as well as to systemic lupus erythematosus (SLE).

Objectives: The aim of the study was to evaluate the most common NVC pictures in a population of UCTD patients and if selected NVC pictures might be linked to SLE onset in these patients.

Methods: We evaluated a cohort of 42 UCTD-affected women, diagnosed according to 2014 criteria proposed by Mosca et al. (age, 38 years \pm 46 months; duration of disease, $71 \pm 54$ months) presenting Raynaud's phenomenon. During the observational period (3 years), all of the UCTD patients were evaluated every 6 months. We considered the following NVC parameters/pictures: presence of ectasic capillary loops (diameter $\geq 20 \mu \mathrm{m}$ ); giant capillaries (diameter $\geq 50 \mu \mathrm{m}$ ); hemosiderin deposits/microhemorrages; capillary number reduction; meandering capillaries (tortuosity); elongated capillaries; ramified/bushy capillaries; micro-vascular array disorganization. SLE diagnosis was posed according to the 2012 SLICC/ACR criteria. Qualitative variables were expressed in frequencies; their association, by non-parametric tests; quantitative variables, by analysis of co-variance. Results: Non-specific NVC alterations (for instance, not suggestive of SDD) were detected in $40(98 \%)$ of the UCTD patients during the observational period. On the other hands, the presence of hemosiderin deposits, ectasic loops, elongated and ramified capillaries was found associated to the clinical subgroup of UCTD patients that later developed SLE (4/42 subjects, $10 \%$; $O R=10.5)$. In particular, the independent variables "hemosiderin deposits/microhemorrages" (OR=8.32) and "elongated capillaries" (OR 6.28), were found significantly linked to the SLE onset $(p<0.05)$, whereas the independent variables "tortuosity" $(O R=12.16)$ and "ramified/bushy capillaries" (OR 9.47) were, at the opposite, predictive for the prosecution of the status of UCTD patient $(p<0.05)$.

Conclusions: The present study reports NVC pictures that can be more frequently observed in UCTD patients that include "tortuosity" and "ramified/bushy capillaries". In addition, the NVC analysis suggests the presence of typical capillaroscopic microvascular abnormalities, "hemosiderin deposits/microhemorrages" and "elongated capillaries", that more frequently seem observed in those UCTD patients that move to SLE onset.

References:

[1] Hughes M et al. Best Pract Res Clin Rheumatol. 2016;30:112-32.

[2] Ingegnoli F, et al. J Clin Rheumatol. 2005;11(6):295-8.

[3] Mosca M, et al. J Autoimmun. 2014:48-9:50-2.

[4] Cutolo M et al. Best Pract Res Clin Rheumatol. 2005;19(3):437-52.

Disclosure of Interest: None declared

DOI: 10.1136/annrheumdis-2017-eular.5742

\section{FRI0289 CEREBROVASCULAR DISEASE IN THE ANTIPHOSPHOLIPID SYNDROME}

M. Estévez, A. Argibay, L. Rodriguez, M. Freire, B. Gimena,

J. Fernández-Martín, A. Rivera. Systemic Autoimmune diseases and Thrombosis Unit, University Hospital Complex of Vigo, Vigo, Spain

Background: Antiphospholipid syndrome (APS) is a thrombophilic disorder characterized by recurrent arterial and venous thrombosis, and also pregnancy losses associated to antiphospholipid antibodies (APA). Cerebrovascular disease (CVD) is the most common and severe arterial thrombotic manifestation in patients with APS.

Objectives: 1.To determine the prevalence and the type of CVD in patients with APS. 2.To compare the recurrent strokes, affected brain areas, hospitalization, treatment and mortality between patients with CVD, with and without APS. 